\title{
Study on the Aerodynamic Characteristics of Tunnel and Metro Station with PSD During High-speed Train Passing Tunnel
}

\author{
Luo Jian-jun ${ }^{1, \text { a }}$ \\ ${ }^{1}$ Tunnel and Underground Engineering Research Center of Ministry of Education, \\ Beijing Jiaotong University, Beijing, China, 100044 \\ ${ }^{a}$ Beijing's Key Laboratory of Structural Wind Engineering and Urban Wind \\ Environment; School of Civil Engineering, Beijing Jiaotong University, Beijing P. \\ R. China. 100044
}

\begin{abstract}
A numerical simulation on the 3D compressible viscous unsteady flow field induced by a high-speed subway train, while it's passing by the platform screen door of subway station, is presented in the paper. The research on transient pressure in tunnel due to changes of positions of platform screen door is proceeded in order to design platform screen doors for underground stations of Dong-guan to Huizhou inter-city rail traffic. Result shows that the platform is subjected to aerodynamic compression and tension cycle loads all the time. And compression load is larger than tension load. The maximum and minimum pressure values in absolute terms got from measuring points of the whole station decrease gradually along the direction train going, no matter whether the platform screen door is fixed or not. The absolute values of maximum and minimum pressure of piston wind generated at platform screen door are decreasing while the spacing between platform screen door and platform becomes wider. The pressure value of air at exit is larger than that of air at entrance. As for a high-speed subway station, it is a practicable way to set air shafts at entrance and exit of station to mitigate pressure of air in station and tunnel. The pressure value of air could have a 10 to 15 percent reduction if there is an air shaft. Besides, the platform screen door can be installed in half tall of PSD.
\end{abstract}

Keywords: High-speed train; Aerodynamic; Compression wave; PSD; Ventilation Shaft

\section{Introduction}

With the development of traffic, there are coming more demands for transport, like high-speed, efficient work, environmental protection and so on. So people believe that high-speed rail connection of big cities will become the preferred mode of transport. Owing to a shortage of urban land gradually, building high-speed railway station underground has become a new trend in the development of railway transportation.

As the train at high speed running into and passing through the tunnel and underground station, there will be an obvious fluctuation of pressure produced in the tunnel and station, which could affect the passenger comfort and tunnel surrounding environment, and also bring safety concerns of equipment within the station at the same time. With the raising of train speed, the aerodynamic effect will be more and more significant. When a high-speed trains passes through the underground railway station (don't staying), there would appear many factors, no matter on line or platform, leading to a series of aerodynamic problems and safety risk of passengers and crew. It is also a crucial problem to be solved in the process of high speed railway.

Platform screen door system was an advanced device and was first used in the subway station in 1980s. The subway platform screen door system can greatly improve the safety and comfort of passengers, and had a good effect in saving energy. It was first used in Guangzhou metro line 2 in China and performed excellently. It will be also used in the 
construction and renovation of the subway station in the future, such as the Beijing metro line 4,5 .

The researches on barrier door of subway station and tunnel mainly focus on the design of the barrier door itself and environment control. Zhou Ru made a study of the influence of barrier door on gas diffusion in the fire flue of platform layer, and analyzed the effects and control of flue gas diffusion in platform layer of 2 typical structures-island type and side type [1-2]. Zheng Jinli analyzed the effects of barrier door on air environment of station[3]. Chen long had summarized and analyzed the factor of barrier door on the leakage of air in metro platform [4]. Li Peng combined the advantages of shield door and opened type system with considering in transition season by using numerical simulation, and study shows that train piston wind would be fully brought by installing controllable air outlet on the barrier door for more ventilation between station and outdoor. Li also analyzed velocity field and temperature field of station and finally judged that this new model has a certain effect in saving energy [5].Zhang jie obtained systematic and practical reliability index by calculating, and proposed relevantly suggestions about redundant design, quota design, electromagnetic compatibility design, selection of components, and the reliable measures of the operation and maintenance [6].Han Huaxuan simulated the aerodynamic effects of barrier doors in the underground stations on different working conditions, and analyzed the peak pressure and the rule of pressure fluctuation curve in test results, and finally concluded the influence rule of the pneumatic effect of barrier door on underground station [7].He Lei analyzed the rule of air diffusion in subway stations flue when barrier door was open[8].Zhang Pei-hong tested and analyzed velocity field in platform of subway station which used overall height and non-close barrier door[9]. Yang Wei-chao analyzed the aerodynamic characteristics of the metro shielding door under the action of the piston wind, studied the pressure change mechanism and the change of time and space features, and analyzed the relationship of screen door pressure and train running speed, blocking ratio, area of the piston wind well, the wind valve state, tunnel ventilation scheme and many other factors. Results show that the shielding door pressure changes mainly due to two factors - the pressure of the train Walcott turbulence and tunnel pressure wave [10].

Hui-Guan intercity rapid rail transit line, whose maximum design speed is $140 \mathrm{~km} / \mathrm{h}$ (the forward speed is $200 \mathrm{~km} / \mathrm{h}$ ), is the highest speed city rapid railway in our country at present .In this paper, we will apply fluid software Fluent to simulate Hui-Guan intercity rail transit project, which forward design speed is $200 \mathrm{~km}$ per hour, and consider the influence of aerodynamic load on platform screen door in the worst situation to study the pneumatic pressure change rule when the high-speed train operate and run through the station as the screen door closing. At last we will evaluate and analysis the results to provide reference for the Hui-Guan underground station shielding door design.

\section{Control Equation}

Under the coordinates of Cartesian, the unsteady compressible continuous equation, Reynolds average $N-S$ equation and standard $k-\varepsilon$ model tensor form are as follows:

$$
\begin{aligned}
& \frac{\partial \overline{U_{i}}}{\partial x_{i}}=0 \\
& \frac{\partial k}{\partial t}+\overline{U_{j}} \frac{\partial k}{\partial x_{j}}=\frac{\partial}{\partial x_{j}}\left(\frac{v_{t}}{\sigma_{k}} \frac{\partial k}{\partial x_{j}}\right)+v_{t}\left(\frac{\partial \overline{U_{i}}}{\partial x_{j}}+\frac{\partial \overline{U_{j}}}{\partial x_{i}}\right) \frac{\partial \overline{U_{i}}}{\partial x_{j}}-\varepsilon
\end{aligned}
$$


$\frac{\partial \varepsilon}{\partial t}+\overline{U_{j}} \frac{\partial \varepsilon}{\partial x_{j}}=\frac{\partial}{\partial x_{j}}\left(\frac{v_{t}}{\sigma_{\varepsilon}} \frac{\partial \varepsilon}{\partial x_{j}}\right)+\frac{\varepsilon C_{\varepsilon 1} v_{t}}{k}\left(\frac{\partial \overline{U_{i}}}{\partial x_{j}}+\frac{\partial \overline{U_{j}}}{\partial x_{i}}\right) \frac{\partial \overline{U_{i}}}{\partial x_{j}}-\frac{\varepsilon^{2} C_{\varepsilon 2}}{k}$

$v_{t}=C_{\mu} \frac{k^{2}}{\varepsilon}$

$\overline{u_{i} u_{j}}=-v_{t}\left(\frac{\partial \overline{U_{i}}}{\partial x_{j}}+\frac{\partial \bar{U}}{\partial x_{i}}\right)+\frac{2}{3} k \delta_{i j}$

Respectively, ${ }^{x_{i}}$ is Cartesian coordinate component,${ }^{t}$ is time, ${ }^{\bar{U}}{ }^{i}$ and ${ }^{u_{i}}$ refers to the average velocity and fluctuating velocity, $\bar{p}$ is the average pressure, $\rho$ refers to the density, ${ }^{v}$ refers to the kinematic viscosity coefficient, ${ }^{v_{t}}$ refers to the turbulent viscosity, $g_{i}$ is gravity acceleration component, ${ }^{k}$ is the turbulent kinetic energy, $\varepsilon$ is the turbulent kinetic energy dissipation rate, ${ }^{\delta_{i j}}$ is the kronecker delta. In the $k-\varepsilon$ model above, the constant experience values are as follows:

$$
C_{\mu}=0.09, C_{\varepsilon 1}=1.44, C_{\varepsilon 2}=1.92, \sigma_{k}=1.0, \sigma_{\varepsilon}=1.3 \text {.Control equations are }
$$
discretely solved by finite volume method, PISO algorithm is used to solve the problem of pressure and velocity coupling. The second order format is used for the wind convection discretization of diffusion. PRESTO is used for discrete pressure correction equation. In the analysis of the unsteady flow, the time derivative of the first-order implicit method is discrete.

\section{Basic Conditions and Calculation Method}

Calculating basic conditions as shown in Table 1.

Table 1. The Basis Conditions of Calculation

\begin{tabular}{cc}
\hline Train head parameters $\left[\mathrm{m}^{2}\right]$ & CRH2,S=11.4 \\
\hline The length of the train[m] & Eight marshalling,200.04 \\
The train speed $[\mathrm{km} / \mathrm{h}]$ & 200 \\
The length of the tunnel[m] & 2145,2000 \\
Tunnel cross section shape $[\mathrm{m}]$ & Round [shield tunnel] \\
Length of the station $[\mathrm{m}]$ & 313 \\
Tunnel sectional area $\left[\mathrm{m}^{2}\right]$ & 50.48 \\
Platform screen door and the platform edge $[\mathrm{m}]$ & $0,0.5,1.0,1.2,1.5$ \\
Space area formed by screen door and roof & sidewall $\left[\mathrm{m}^{2}\right]$ \\
Measuring point location & $28.02,31.36,34.52,35.82,37.77$ \\
\end{tabular}




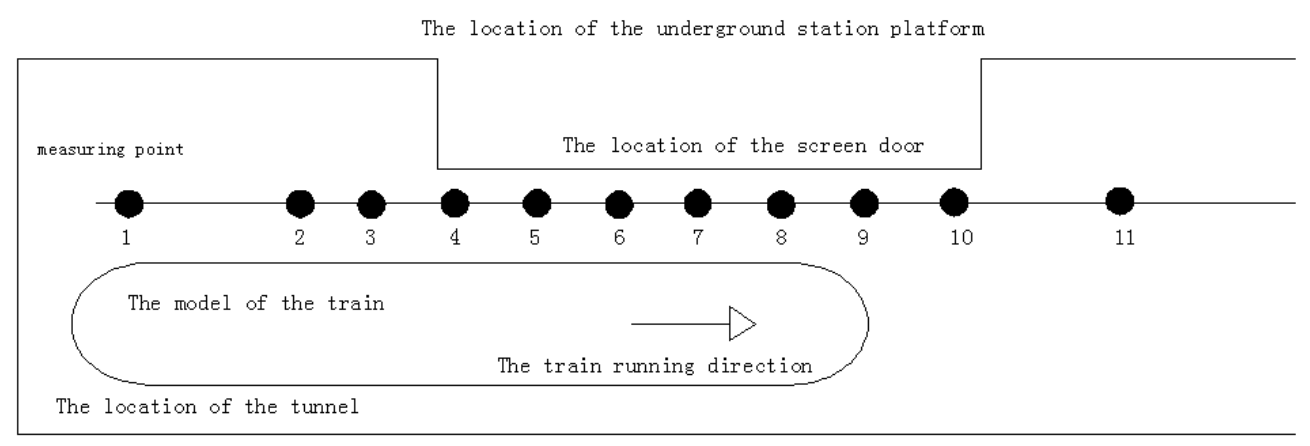

Figure 1. The Measuring Points Plan Diagram of Tunnels and Station

Because this paper studies the situation when the train passes through the station, so we chose two ranges and a station as the calculation area, the length of the station and regional tunnel as shown in table 1.The total length of calculation area is $2145+2000+500+500$ $=5145 \mathrm{~m}$. The size of the station is under consideration only when the screen door is closed and the cross section area is made of tunnel roof and side wall.

In subway tunnel boundary conditions setting, velocity on the surface of the tunnel and side of the train is zero, secondly the normal component of the train surface pressure gradient is zero. Because this article only analyzes the aerodynamic effect of the station area on both ends and the screen door location, so we chose the location of tunnel entrance and the export of the next tunnel as the far field boundary of the calculation area, with no reflection boundary condition based on Riemann invariants, namely, the compression wave propagates out and reaches the surface of the calculation area without reflection.

The length of iteration step and time step in the process of numerical calculation have great influence on convergence of the calculation results, the iteration step of the calculation is 50 , time step ${ }^{\Delta_{t}}$ takes $0.0001 \mathrm{~s}$. As to the verification of the calculation model for the paper, we can refer to the literature [11-12].

For the realization of the relative movement between the train and the tunnel, the UDF custom dynamic grid technique, and simplify CRH2 railways train wheels, motors, track bed and track rail are applied. Because we need capture the compression wave in calculation, the calculation grid isn't allowed to be too small, the calculation area on both ends of the tunnel and the station which have mutated parts with dense mesh. The calculation grid of tunnel entrance and tunnel exit minimum size is $0.1 \mathrm{~m}$, and tunnel compression wave in a certain area of tunnel characterized by plane wave, we can adopt $\mathrm{H}$ type hexahedral grid. There are 45049127 tetrahedron and hexahedron grid mixing units after dividing grid to the whole calculation area. Part grid of the calculation area as shown in figure 2.Due to the great number of grid division of calculation, the method of parallel computing is used. 


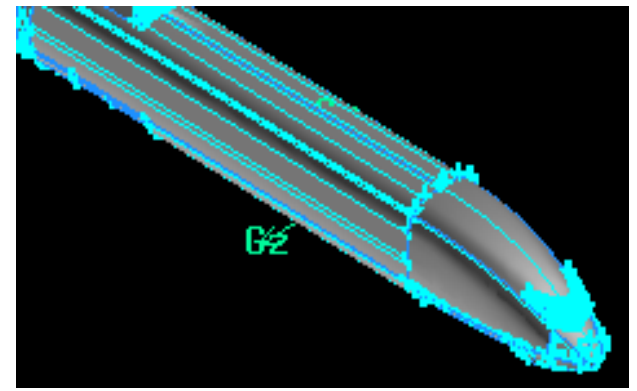

the model of $\mathrm{CRH} 2$ train head

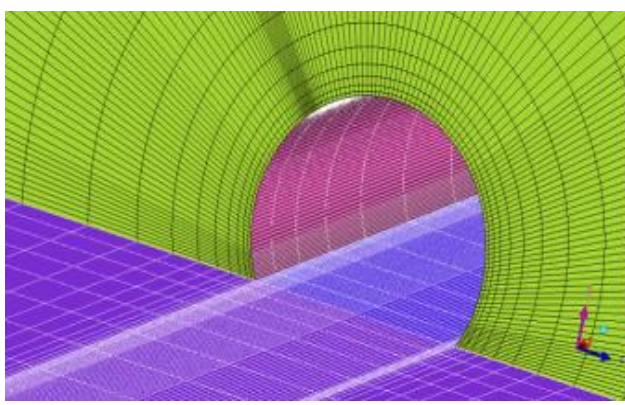

entrance of single track tunnel model

Figure 2: The Mesh of the Train and the Entrance of Single Track Tunnel Model

\section{The Calculation Results and Analysis}

\subsection{The Effect of Pressure Changes in Tunnel Pulling Section}

Figure 3 shows that changes of the distance between shielding door and the platform edge changed the cavity area formed by shielding door and tunnel roof and side wall. Because the measuring point of tunnel is far away from the station, it have no effect on the measuring point 1 of the first region which is away from the station entrance.

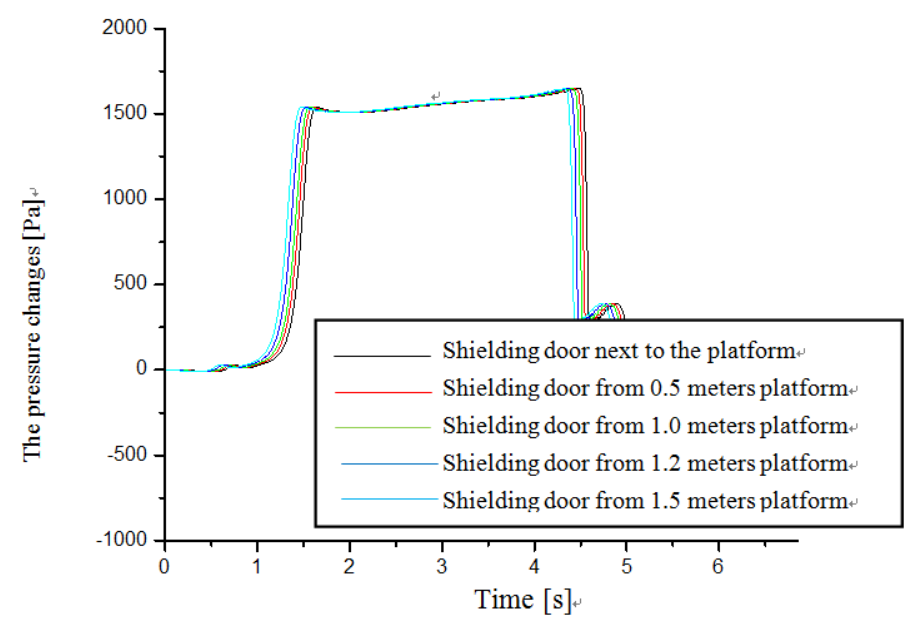

\section{Figure 3. Pressure History for Measuring Point One in Tunnel (1000m from the Station Entrance)}

Figure $4 \sim 5$ is the pressure history for measuring points in tunnel which are near by the tunnel and station connecting parts (100m from the station entrance) when the screen door position changed. In the first tunnel, when the compression wave generated by the train arrived in tunnel and station connection parts, because the connection parts section change suddenly(area from large to small)and gas density rises, it leads to pressure of measuring point close to joint position of the station increasing gradually. In the tunnel, while measuring point is closer to the station entrance, pressure rises more rapidly. Secondly, as the screen door location being away from the station platform edge, measuring point pressure gradually decline, and it was considered that when screen door is away from the platform edge, cavity area formed by screen door, the tunnel roof and floor and wall is expanding, which is advantageous to the pressure in the tunnel "leaking". But in the Figures 4 and 5 there are still differences in the rising period of pressure curves: while pressure curve of Figure 4 has two rising stage, the one of Figure 5 has a rising stage and a decline 
stage. It could be explained that when the points is closer to the connection part of the tunnel and station, there was a whirl vortex long formed in the region, which leads to the pressure declining.

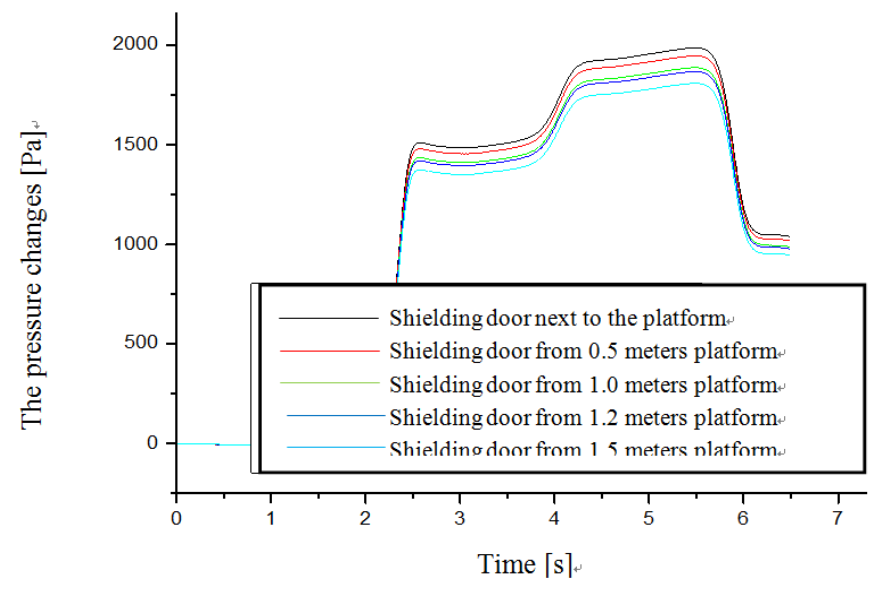

Figure 4. Pressure History for Measuring Point Two in Tunnel (100m from the Station Entrance)

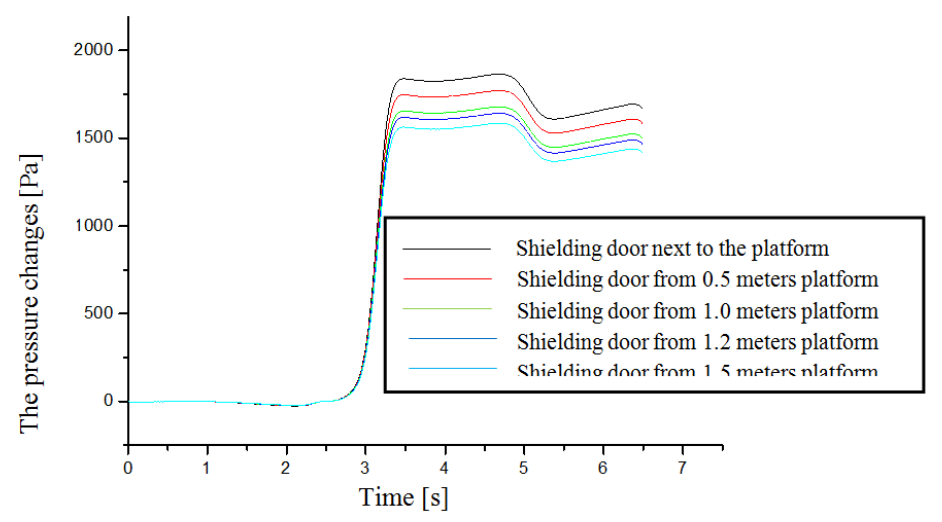

Figure 5. Pressure History for Measuring Point Three in Tunnel (50m from the Station Entrance)

\subsection{Pressure Changes in Tunnel Cavity that Within the Scope of Station Screen Door Section}

Figure $6 \sim 8$ is the pressure change curve of the measuring point in the tunnel when the distance from screen door to platform edge changes. Calculation results show that with the distance between the screen door and the platform edge increasing, the space sectional area formed increases and when the screen door is closed, the pressure is reduced each measuring point, and showed nonlinear changes. Time history curve of three measuring points above indicates that the change in distance between the screen door and platform at the same measuring point $(\mathrm{L})$, the platform position, and the change trend of time history curve are consistent at those three measuring points. 


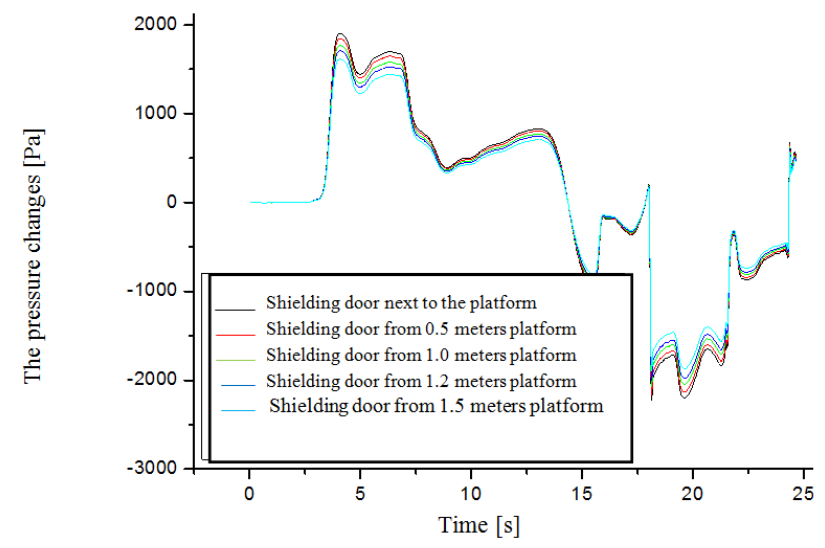

Figure 6. Pressure History for Measuring Point in Station (10m from the Station Entrance)

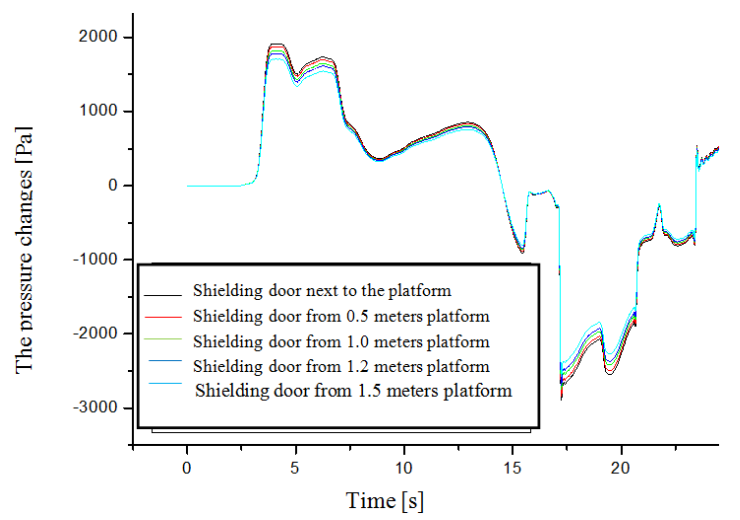

Figure 7. Pressure History for Measuring Point in Station (150m from the Station Entrance)

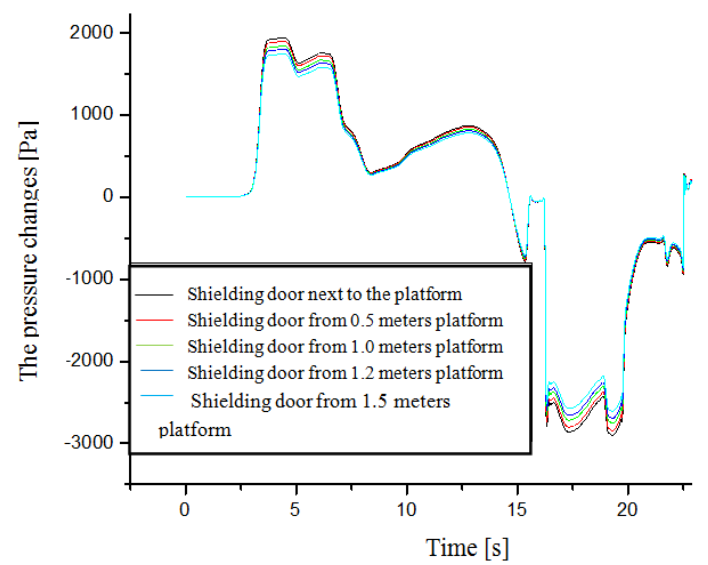

Figure 8. Pressure History for Measuring Point in Station(300m from the Station Entrance) 


\subsection{Pressure Changes in Station Exit Section Tunnel}

Figure 9 show that with the distance between the screen door and the platform edge increasing, the cavity sectional area formed by the screen door ,the tunnel roof , floor and wall increases, and the pressure of each measuring point in the next tunnel decreases. About 20m from the exit (the location of the next tunnel inlet), pressure curve appeared two peaks. The first peak is due to the train into the first tunnel, the second is due to the first wave arrived at the connection part, because of mutations (area changed from small to big), compression wave occurs reflection phenomenon in here, thus compression wave and reflected wave keep occurring wave superposition, which leads to the second peak occurs.

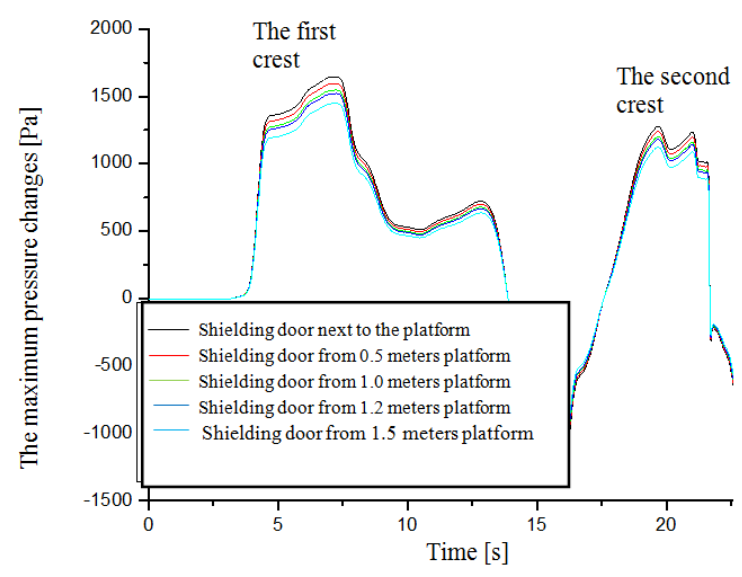

Figure 9. Pressure History for Measuring Point in Station(20m from the Station Exit)

\subsection{The Maximum Regulation of Pressure Change at Each Measuring Point Within the Scope of the Station}

According to the previous Figure 3 to Figure 9, we can know that each measuring point in the process of history shown the maximum value and the minimum value. That is to say, the station platform screen door is affected by compression wave and expansion wave when the train runs through the station, which means that screen door withstands compression and tensile force.The designer must consider that the screen door is influnced by compression and tensile cyclic loading when they are designing the strength of the screen door. Figure 10 shows that as for a certain measuring point, the maximum positive pressure of the screen door decreases as the distance between screen door and platform increasing, while the maximum negative pressure of the screen door increases as the distance increasing. But the absolute value of the negative pressure is larger than the maximum positive value. 


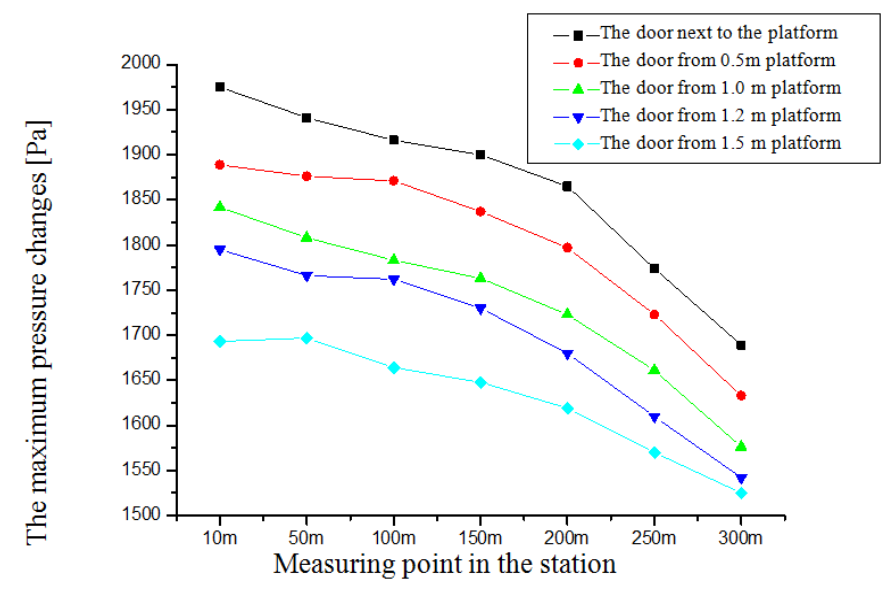

Figure 10. Relationship Between Max-Pressure of MeasuringP on PSD and Distance from PSD to Platform of Metro

As Figure 11 shows, when the position of screen door and platform edge fixed, from the station inlet end to the station exit end, as the screen door location away from the edge of the platform, the absolute minimum value at measuring point within the scope of the entire station also reduced gradually.

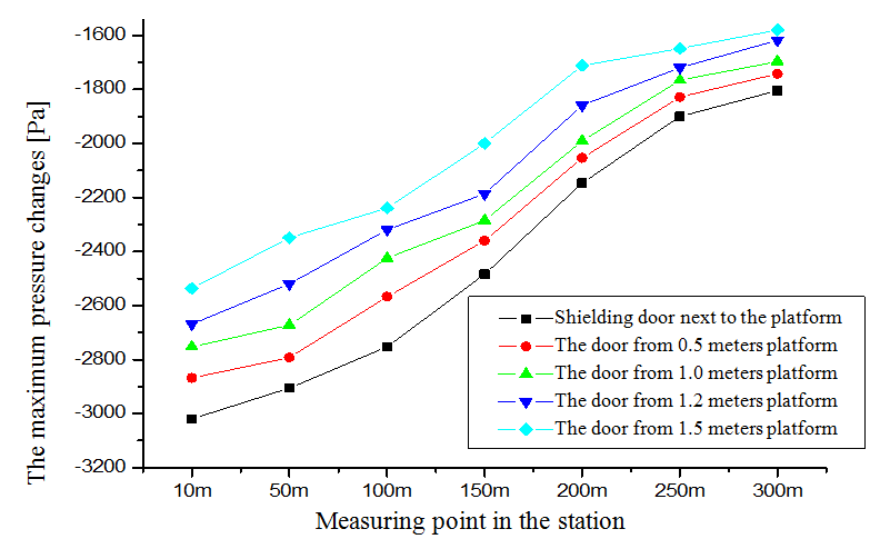

Figure 11. Relationship Between Min-Pressure of Measuring Point on PSD and Distance from PSD to Platform of Metro

Figure 12 shows the change law of maximum pressure within the tunnel at the ends of the station and the tunnel at the entire platform screen door. The calculation results show that:

(1)In the station inlet tunnel: along the direction of the train, away from the station within a certain range, the maximum pressure of the tunnel inner measuring point basically remains unchanged, but it gradually increases near to the station entrance.

(2)The maximum pressure at the measuring point within the scope of the station decreases, especially at the end the station, and due to the next tunnel sectional area increases, air depression area within the scope of the station is increasing. Thus the reduction amplitude of maximum pressure is comparatively large in the end of the station, but in the station entrance it is relatively small.

(3)In the tunnel, from the outlet of the station to the export, the maximum pressure decreases gradually.

(4)From the point of various working conditions, the maximum pressure at the measuring point at the interface of the station exit and the next tunnel is larger than the ones at the first tunnel far away from the scope of the station. Because within the scope of 
the whole range + the station, there is a section area shrinking so that the maximum pressure has a rising stage in a narrow range.

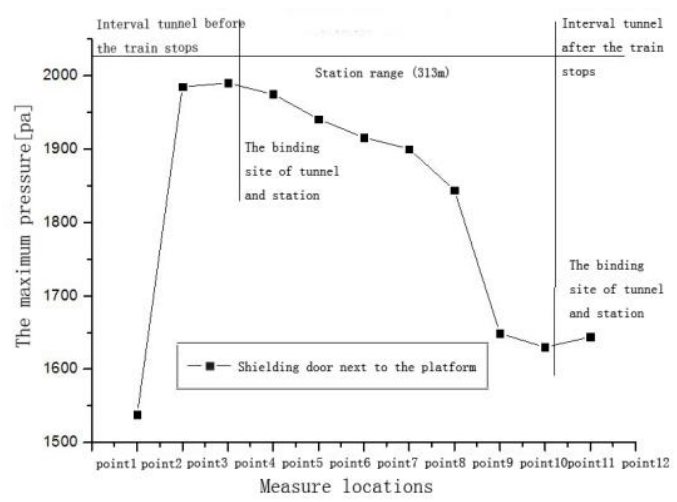

Figure 12. Relationship Between Max-Pressure of Measuring Point on PSD and Location on the Edge of the Metro Platform

\subsection{Change of the Piston Wind at the Location of Station Screen Door.}

Figure 13 is the variation of maximum and minimum values of piston wind when the distance from the screen door to the edge of the platform changes, at the measuring point (10 $\mathrm{m}$ from the station entrance end) at the location of the screen door. As Figure 13 shows, because the tunnel section area at the location of the screen door is relatively small, the results in the tunnel blockage ratio becomes relatively large, which causes stronger piston wind produced by train, and the highest train wind speed can reach $35 \mathrm{~m} / \mathrm{s}$.Secondly, with the distance between screen door and the edge of the platform increasing, the absolute value of the maximum and minimum are both reduced.

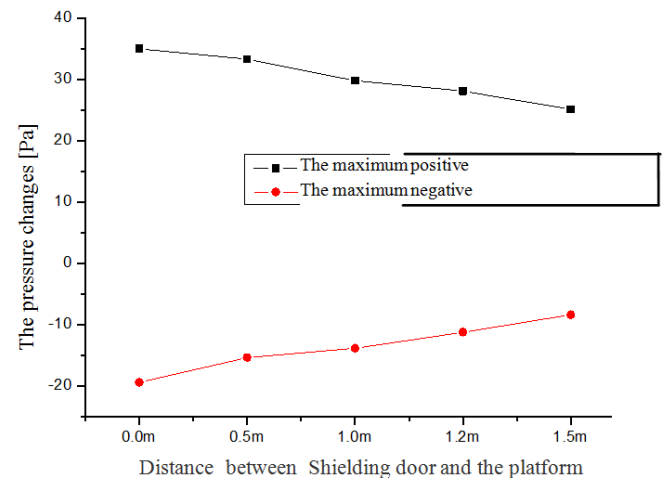

Figure 13. The Change of Maximum Wind Speed of PSD at 10m Away from the Station EntranceA the $+Z$ Direction

\subsection{The Decompression Effect of Air Vents on Both Ends of the Station}

According to the requirements of designer, one ventilation shaft is located at the station top and 10m away from the station entrance, and aims at slowing down the impact effect of compression wave caused by train on the screen door, and prolonging the life of the screen door. The section area of the shaft is $12.25 \mathrm{~m} 2$. Figure $14 \sim 16$ are the comparisons of influence between ventilation shafts and no shaft at different measuring points. Results of calculation show that when there is a ventilation shaft, the pressure at the screen door reduces in a certain degree, and the reduction is about $10 \sim 15 \%$. 


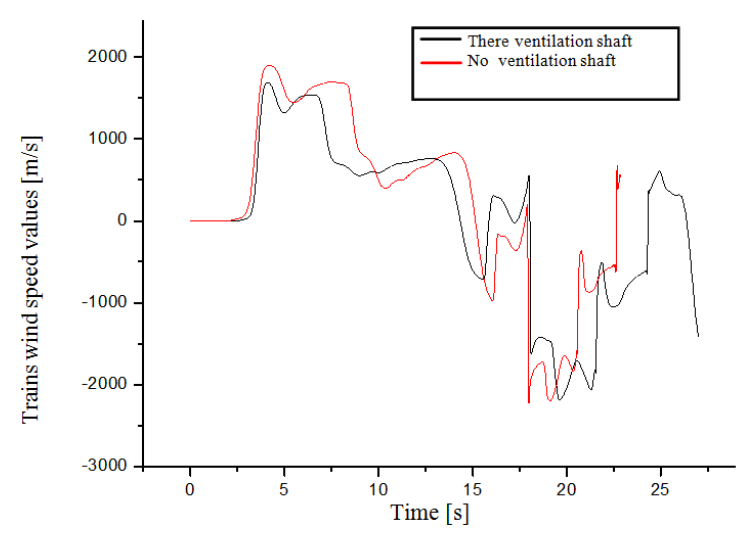

Figure 14. The Curve of Pressure Change with and Without Ventilation Shafts (10m from the Station Entrance)

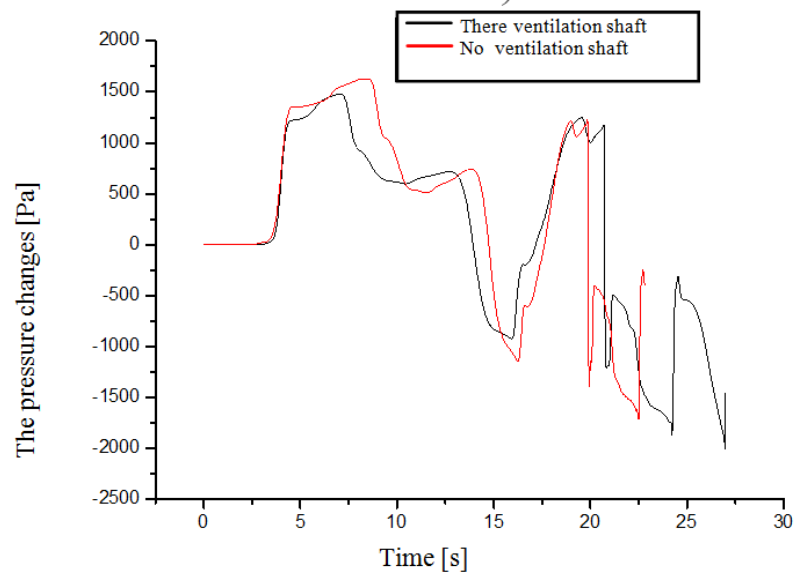

Figure 15. The Curve of Pressure Change with and Without Ventilation Shafts (150m from the Station Entrance)

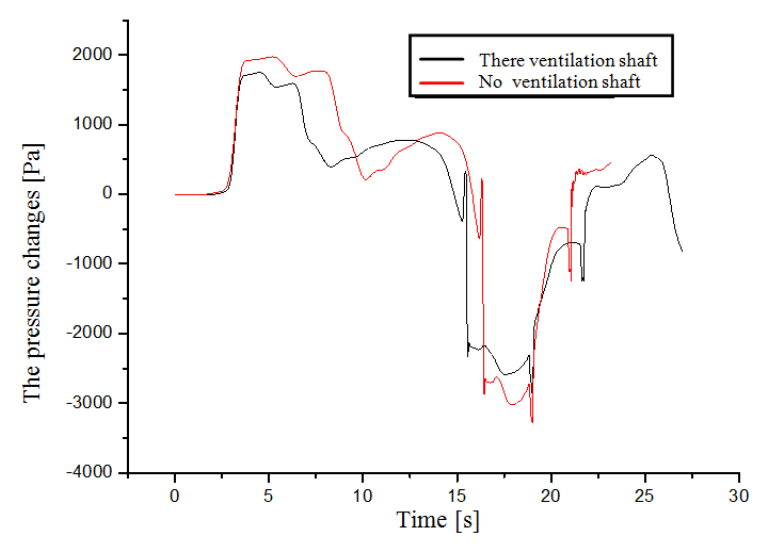

Figure 16. The Curve of Pressure Change with and Without Ventilation Shafts (300m from the Station Entrance) 


\section{Table 2. The Pressure Comparison of Measuring Point on Safety Screen Door with and Without Ventilation Shafts}

\begin{tabular}{|c|c|c|c|c|}
\hline \multirow{2}{*}{$\begin{array}{l}\text { Location of } \\
\text { measuring point }\end{array}$} & \multicolumn{2}{|c|}{ pressure without ventilation shafts $[\mathrm{Pa}]$} & \multicolumn{2}{|c|}{ pressure with ventilation shaft $[\mathrm{pa}]$} \\
\hline & $\begin{array}{c}\text { Maximum } \\
\text { pressure }\end{array}$ & $\begin{array}{c}\text { Minimum } \\
\text { pressure }\end{array}$ & $\begin{array}{c}\text { Maximum } \\
\text { pressure }\end{array}$ & $\begin{array}{c}\text { Minimum } \\
\text { pressure }\end{array}$ \\
\hline $10 \mathrm{~m}$ & 1975 & -3214 & 1750 & -2820 \\
\hline $50 \mathrm{~m}$ & 1941 & -2889 & 1740 & -2747 \\
\hline $100 \mathrm{~m}$ & 1916 & -2894 & 1717 & -2435 \\
\hline $150 \mathrm{~m}$ & 1900 & -2232 & 1691 & -2169 \\
\hline $200 \mathrm{~m}$ & 1844 & -2162 & 1611 & -2379 \\
\hline $250 \mathrm{~m}$ & 1649 & -2000 & 1496 & -2120 \\
\hline $300 \mathrm{~m}$ & 1630 & -1716 & 1477 & -1922 \\
\hline
\end{tabular}

\section{The Conclusions and Suggestions}

(1) when the position of screen door was fixed, the maximum pressure at measuring point within the scope of the station gradually reduced, especially near the end of the station. And due to the next tunnel sectional area increases, the air depression area within the scope of station is increasing. Thus the reduction amplitude of maximum pressure is comparatively large in the end of the station, but in the station entrance it is relatively small.

(2) In the process of high-speed metro train passing through the station, screen door is subjected to compression and tensile cyclic aerodynamic load, and the maximum tensile effect is larger than the maximum compression effect.

(3) Whether the position of screen door is fixed or not, the absolute value of the minimum at the measuring point within the scope of the entire station would decrease.

(4) While the distance between the screen door and the edge of the platform increases, the absolute value of the maximum and minimum are both reduced.

(5) For the underground station, setting the ventilation shafts could reduce the pressure of the station and the tunnel, which is a kind of feasible mitigation measure. In this paper, setting a ventilation shaft can reduce the pressure $10 \sim 15 \%$. At the same time, it is possible to go on further optimization, including the location of the ventilation shafts, the size of the sectional area, the number of ventilation shaft, etc.

(6) This paper made an analysis on load conditions of the screen door when the train passes through the station .To check out the security problem of the structure of the screen door, the related manufacturers of screen door are suggested to do related calculations or experiments on compression - tensile load test, which could test the fatigue strength and predict the safety life of the screen door. Secondly, in order to reduce the effect of pneumatic cyclic loading on the structure of the screen door, it is suggested that the subway operation units can take half high screen door into consideration when screen doors are installed.

\section{Acknowledgments}

The author wishes to acknowledge the support and motivation provided by National High-tech R\&D program of China (No. 2011AA11A103), and National Natural Science Foundation of China (No. 51178030), and the Fundamental Scientific Research Funds of Beijing Jiaotong University (No. 2011JBM078). 


\section{References}

[1] R. Zhou, J. He and J. Jiang, "Effects of the PED on Smoke Control of Subway Station Fires", Journal of the China Railway Society, vol. 30, no. 5, (2008), pp. 62-67.

[2] R. Zhou, J. He and J. Jiang, "Smoke Diffusion and Control at Different Platform Floor Structure of Subway Station in Fire", China Railway Science, vol. 29, no. 06, (2008), pp. 126-131.

[3] Z. J. Li, "The environmental effect of PSD to Underground Station", Underground Engineering and Tunnels, no. 4, (2008), pp. 46-48.

[4] L. Chen and C. J. Zhao, "Calculation and Analysis for the Leakage Air Flow Rate of Station Screen Door", Science Technology and Engineering, vol. 23, no. 9, (2009) December, pp. 2730-2732.

[5] L. Peng, F.Lian and C. Li, "Numerical simulation of the controllable tuyere on metro PSD system", Refrigeration and Air Conditionin, vol. 24, no. 1, (2010) Febuary, pp. 76-78.

[6] J. Zhang, "Reliability Design of Metro Platform Screen Doors", vol. 310, no. 23, (2009) January, pp. 89-190.

[7] H. X. Han and W. Yingxue, "Study on the Effect of Aerodynamic for PSD to High-Speed Railway Underground Station", Railway Standard Design, vol. 1, (2008), pp. 108-110.

[8] L. He, B. Lei and H.Q. Bi, "Analysis of air flow regularity in metro station during opening of protective door", Journal of Railway Engineering, no. 12, (2013), pp. 39-42.

[9] P.-H. Zhang, S. Wang and R.-X. Shang, "Test and analysis on the velocity field of subway station with full-height non-enclosed screen doors", Journal of Safety Science and Technology, vol. 8, no. 9, (2012), pp. 22-26.

[10] W. C. Yang, L. M. Peng and C. H. Shi, "Analysis of aerodynamic characteristics of PSD under the piston wind influence in subway", Journal of Zhengzhou University, vol. 30, no. 2, (2009), pp. 120-124.

[11] J. J. Luo, B. Gao and Y. X. Wang, "Numerical simulation of unsteady three-dimensional flow induced by high-speed train entering tunnel with shaft", Journal of Southwest Jiaotong University, vol. 39, no. 4, (2004), pp. 442-446.

[12] J. J. Luo, B. Gao, Y. X. Wang and L. G. Li, "Numerical Study on the Pressure Change when High-speed Train Passing Through a Tunnel", Journal of China Railway Science, vol. 24, no. 4, (2003), pp. 82-86.

\section{Author}

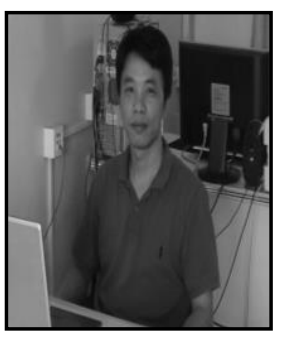

Luo Jianjun, Beijing Jiaotong University, Associate Professor. 
International Journal of Signal Processing, Image Processing and Pattern Recognition Vol. 9, No. 6 (2016) 